\title{
VECTOR PERTURBATION PRECODING FOR RECEIVERS WITH LIMITED DYNAMIC RANGE
}

\author{
Johannes Maurer $^{\star}$, Joakim Jaldén ${ }^{\star}$, Dominik Seethaler ${ }^{\diamond}$, and Gerald Matz ${ }^{\star}$ \\ * Institute of Communications and Radio-Frequency Engineering, Vienna University of Technology \\ Gusshausstrasse 25/389, A-1040 Vienna, Austria (Europe), phone: +43 158801 38968; E-mail: jmaurer@nt.tuwien.ac.at \\ $\diamond$ Communication Technology Laboratory, ETH Zürich \\ Sternwartstrasse 7, CH-8092 Zürich, Switzerland, phone: +41 4463 25937; E-mail: seethal@ nari.ee.ethz.ch
}

\begin{abstract}
In this paper we consider the vector perturbation (VP) precoding scheme for the multiuser MISO broadcast channel proposed by Hochwald et al. under the practical assumption that the receivers have limited dynamic range. In this case, VP precoding is shown to suffer from an error floor at high signal-to-noise ratio (SNR). As an alternative, we propose precoding with restricted VP (RVP), which takes the limited dynamic range of the receivers explicitly into account by restricting to a finite set of possible perturbation vectors at the transmitter side. We derive the diversity order of this RVP scheme and show that no error floor occurs and that the performance is superior to VP for the entire range of SNRs.
\end{abstract}

Index Terms - Multiuser MIMO systems, vector perturbation precoding, diversity order, dynamic range.

\section{INTRODUCTION}

We consider a wireless broadcast scenario in which a base station uses multiple antennas to transmit simultaneously to multiple users. In this context, precoding based on vector perturbation (VP) [1-3] is a promising technique since it enables the users to perform lowcomplexity optimal detection in a non-cooperative manner. This is accomplished by performing channel inversion at the base station, preceded by a perturbation of the transmit vector in order to reduce the transmit power [2]. The receivers detect their data symbols by scaling their receive signal, followed by a modulo operation and scalar symbol detection. Throughout the paper, we will refer to this scheme as conventional VP precoding.

An inherent problem with conventional VP precoding, which has not been considered in detail previously, is the large (or even unbounded) dynamic range of the signals at the detector input. A similar problem is well known in the context of Tomlinson-Harashima precoding for time-dispersive channels [4]. The large dynamic range of the receive signal represents a serious problem for implementations in fixed point arithmetic. This motivates us to consider ways of limiting the dynamic range at the receiver. Our contributions in this paper are as follows:

- We show that conventional VP with a limited dynamic range at the receivers has an error floor in general.

- We propose a precoding scheme based on a finite set of possible perturbation vectors (referred to as restricted vector perturbation (RVP)) to take the limited dynamic range of the receivers into account.

This work is supported by the STREP project MASCOT (IST-026905) within the Sixth Framework Programme of the European Commission.
- We derive the diversity order of the RVP scheme which, although being significantly lower than for unrestricted perturbations, is strictly positive and thus avoids the error floor.

- We show numerical results to support our theoretical findings and we illustrate that the performance loss of RVP as compared to conventional VP (with unlimited dynamic range) can be very small in the relevant SNR-regime.

The paper is organized as follows. Section 2 presents the system model and conventional VP precoding. The impact of receive-side clipping on the performance is analyzed in Section 3. In Section 4, we propose RVP precoding and determine its diversity order. Simulation results and conclusions are presented in Sections 5 and 6, respectively.

\section{BACKGROUND}

\subsection{System Model}

We consider a multi-user communications system operating in the downlink (see e.g. [1,3]). The base station is equipped with $M$ transmit antennas and there are $K$ users, each with a single receive antenna. We assume that $2 \leq K \leq M$ and that there is no user cooperation.

Let $\mathbf{x} \triangleq\left(x_{1} \ldots x_{M}\right)^{\mathrm{T}}$ denote the length- $M$ transmit vector. We assume mutually independent, spatially uncorrelated Rayleigh flat fading MISO channels $\mathbf{h}_{k}, k=1, \ldots, K$, i.e., $\mathbf{h}_{k} \sim \mathcal{C N}\left(\mathbf{0}, M^{-1} \mathbf{I}\right)$. The $k$ th user receives $y_{k}=\mathbf{h}_{k}^{\mathrm{T}} \mathbf{x}+w_{k}$ where the additive noise $w_{k}$ is assumed circularly symmetric complex Gaussian with unit variance. By collecting the receive values of all $K$ users in a receive vector $\mathbf{y} \triangleq\left(y_{1} \ldots y_{K}\right)^{\mathrm{T}}$, the overall channel input-output relation can be written as

$$
\mathbf{y}=\mathbf{H} \mathbf{x}+\mathbf{w}, \quad \text { where } \quad \mathbf{H}=\left(\mathbf{h}_{1} \ldots \mathbf{h}_{K}\right)^{\mathrm{T}}
$$

and $\mathbf{w} \triangleq\left(w_{1} \ldots w_{K}\right)^{\mathrm{T}}$. We assume that the transmit signals are subject to an instantaneous peak power constraint given by

$$
\|\mathbf{x}\|^{2} \leq P
$$

Note that the type of power constraint strongly affects precoding performance [5].

\subsection{VP Precoding}

Transmitter Processing. The base station transmits $K$ (independent) complex symbols $s_{k} \in \mathcal{A}$ simultaneously to the $K$ users, where $\mathcal{A}$ denotes the symbol alphabet. We assume that $0 \notin \mathcal{A}$. Let 
$\mathbf{s} \triangleq\left(s_{1} \ldots s_{K}\right)^{\mathrm{T}}$ denote the data vector obtained by collecting all symbols. With conventional VP precoding [2], the transmit vector is obtained as

$$
\mathbf{x}=\sqrt{\frac{P}{\Gamma(\mathbf{z})}} \mathbf{H}^{\dagger}(\mathbf{s}+\tau \mathbf{z}) .
$$

Here, $\mathbf{H}^{\dagger}=\mathbf{H}^{\mathrm{H}}\left(\mathbf{H} \mathbf{H}^{\mathrm{H}}\right)^{-1}$ denotes the right pseudo inverse, $\tau$ is a fixed real-valued scaling factor, and $\mathbf{z} \triangleq\left(z_{1} \ldots z_{K}\right)^{\mathrm{T}} \in \mathbb{G}^{K}$ is a perturbation vector whose elements are Gaussian integers ${ }^{1}$. The power scaling factor $\Gamma(\mathbf{z})$ is chosen such that the instantaneous power constraint (2) is satisfied with equality, i.e.

$$
\Gamma(\mathbf{z}) \triangleq\left\|\mathbf{H}^{\dagger}(\mathbf{s}+\tau \mathbf{z})\right\|^{2} .
$$

The specific choice of $\mathbf{z}$ will be described later.

Receiver Processing. The $k$ th receiver normalizes the signal power according to

$$
r_{k} \triangleq \sqrt{\frac{\Gamma(\mathbf{z})}{P}} y_{k}
$$

Combining (1) and (3) results in

$$
r_{k}=s_{k}+\tau z_{k}+v_{k}
$$

where $v_{k}=w_{k} \sqrt{\Gamma(\mathbf{z}) / P}$ is white Gaussian noise with variance $\Gamma(\mathbf{z}) / P$. The effective data symbols $s_{k}+\tau z_{k}$ belong to the extended alphabet $\mathcal{A}+\tau \mathbb{G}$ (i.e. all $\tau$-scaled integer translates of $\mathcal{A}$ ). If $\tau$ is selected properly (cf. [2]), each user can perform detection by applying a complex-valued modulo- $\tau$ operation followed by scalar quantization with respect to the symbol alphabet $\mathcal{A}$, i.e.,

$$
\hat{s}_{k}=\mathcal{Q}\left\{\mathcal{M}_{\tau}\left\{r_{k}\right\}\right\},
$$

where $\mathcal{M}_{\tau}\{\cdot\}$ denotes the modulo operation and $\mathcal{Q}\{\cdot\}$ denotes the quantization. Since the noise $v_{k}$ of different users is uncorrelated, this detection is also optimal with respect to the overall probability of error $\operatorname{Pr}\{\mathcal{E}\}=\operatorname{Pr}\{\mathbf{s} \neq \hat{\mathbf{s}}\}$, where $\hat{\mathbf{s}} \triangleq\left(\hat{s}_{1} \ldots \hat{s}_{k}\right)^{\mathrm{T}}$. The detector (6) has very low complexity, which is one of the major advantages of VP precoding.

Optimum Perturbation Vector. In order to minimize the error probability of the detector (6), the SNR in (5) has to be maximized. This amounts to minimizing the noise variance, which equals $\Gamma(\mathbf{z}) / P$. Thus, [2] proposed to choose the optimal perturbation vector $\mathbf{z}$ such that $\Gamma(\mathbf{z})$ in (4) is minimized:

$$
\mathbf{z}^{\star}=\arg \min _{\mathbf{z} \in \mathbb{G}^{K}}\left\|\mathbf{H}^{\dagger}(\mathbf{s}+\tau \mathbf{z})\right\|^{2} .
$$

We will write in the following $\Gamma=\Gamma\left(\mathbf{z}^{\star}\right)$. Since the minimization in (7) is over all Gaussian integers, $\left|z_{k}^{\star}\right|$ can become arbitrarily large. This happens particularly for channel realizations with large condition number since here the "right" direction of $\mathbf{s}+\tau \mathbf{z}^{\star}$ relative to the eigenvectors of $\mathbf{H}^{\dagger}$ is much more important than its length in order for $\Gamma$ to be small. Large values of $\left|z_{k}^{\star}\right|$ in turn result in a large dynamic range of $r_{k}$ in (5).

The minimization problem in (7) is an integer least-squares problem whose complexity in general grows exponentially with the number of users $K$. A promising method to solve (7) is the sphere decoding algorithm [6] (in this context also referred to as sphere encoding [2]), which, however, is still exponentially complex [7] (both in the worst case and on average).

\footnotetext{
${ }^{1}$ The set of Gaussian integers $\mathbb{G}=\mathbb{Z}+j \mathbb{Z}$ comprises all complex numbers with integer real and imaginary parts.
}

\section{RECEIVERS WITH LIMITED DYNAMIC RANGE}

Receiver Model. To model practical receivers where amplifiers, A/D converters, etc. result in limited dynamic range, we let the detector observe a clipped version of $r_{k}$ in (5), denoted $\tilde{r}_{k}$. The symbol decisions are thus obtained according to (6) but with $r_{k}$ replaced by $\tilde{r}_{k}$. We assume that the real and imaginary parts of $r_{k}$ are clipped separately, i.e., $\tilde{r}_{k}=g\left(\operatorname{Re}\left\{r_{k}\right\}\right)+j g\left(\operatorname{Im}\left\{r_{k}\right\}\right)$ where

$$
g(r)= \begin{cases}\kappa \sigma(r), & |r|>\kappa, \\ r, & |r| \leq \kappa,\end{cases}
$$

with $\sigma(\cdot)$ and $\kappa \geq \tau / 2$ denoting the sign function and the clipping threshold, respectively. If the threshold is chosen according to $\kappa=$ $(2 i+1) \tau / 2, i \in\{0,1, \ldots\}$, the clipping does not cut through a periodic repetition of the symbol constellation, thereby avoiding that certain transmit symbols are penalized. We note that clipping may occur both as a consequence of a particularly large perturbation $z_{k}^{\star}$ as well as a large noise realization $v_{k}$. However, the probability of the former will typically outweigh the latter.

Impact on Performance. In this section, we discuss the impact of clipping/limited dynamic range on the performance of conventional VP precoding. Specifically, we establish that for any finite $\kappa$ there is an error floor, i.e., the error probability of the detector has a lower bound that is independent of the SNR. To establish the existence of an error floor, it is sufficient to show that the error probability is bounded away from zero even in the noiseless case. In order to simplify the analysis we restrict attention to 16-QAM or higher constellations since here for any $\kappa$ there exists a symbol $a \in \mathcal{A}$ such that an error is guaranteed if $a$ is transmitted and clipping occurs at the receiver. A sufficient condition for clipping is thus given by

$$
\left|s_{k}+\tau z_{k}^{\star}\right|>\sqrt{2} \kappa
$$

and it follows that

$$
\operatorname{Pr}\{\mathcal{E}\} \geq \operatorname{Pr}\left\{\left|s_{k}+\tau z_{k}^{\star}\right|>\sqrt{2} \kappa \mid s_{k}=a\right\} \operatorname{Pr}\left\{s_{k}=a\right\},
$$

where $\mathcal{E}$ denotes the overall error probability. Since $\operatorname{Pr}\left\{s_{k}=a\right\}>0$ it follows that a sufficient condition for an error floor to occur is given by

$$
\operatorname{Pr}\left\{\left|s_{k}+\tau z_{k}^{\star}\right|>\sqrt{2} \kappa \mid s_{k}=a\right\}>0 .
$$

Although (10) may be established directly, it also follows as a consequence of the discussion in Section 4, and we shall leave the proof for then. In essence, we conclude that the occasional use of large perturbations will lead to an error floor given that the signals in the receiver are subject to a limited dynamic range.

We note that the existence of an error floor may also be established for other constellations, although in this case one must take the direction (in addition to the magnitude) of the perturbation into account. While this is feasible, it is substantially more tedious and we omit the proof due to lack of space.

\section{RVP PRECODING}

In this section, we propose a modification of (7), termed restricted vector perturbation $(R V P)$, which avoids the error floor described in the previous section. Furthermore, we derive the diversity order of RVP precoding with receivers having limited dynamic range.

The main idea is to limit the dynamic range of the receive signals via transmitter processing. Specifically, we propose to use a finite set 

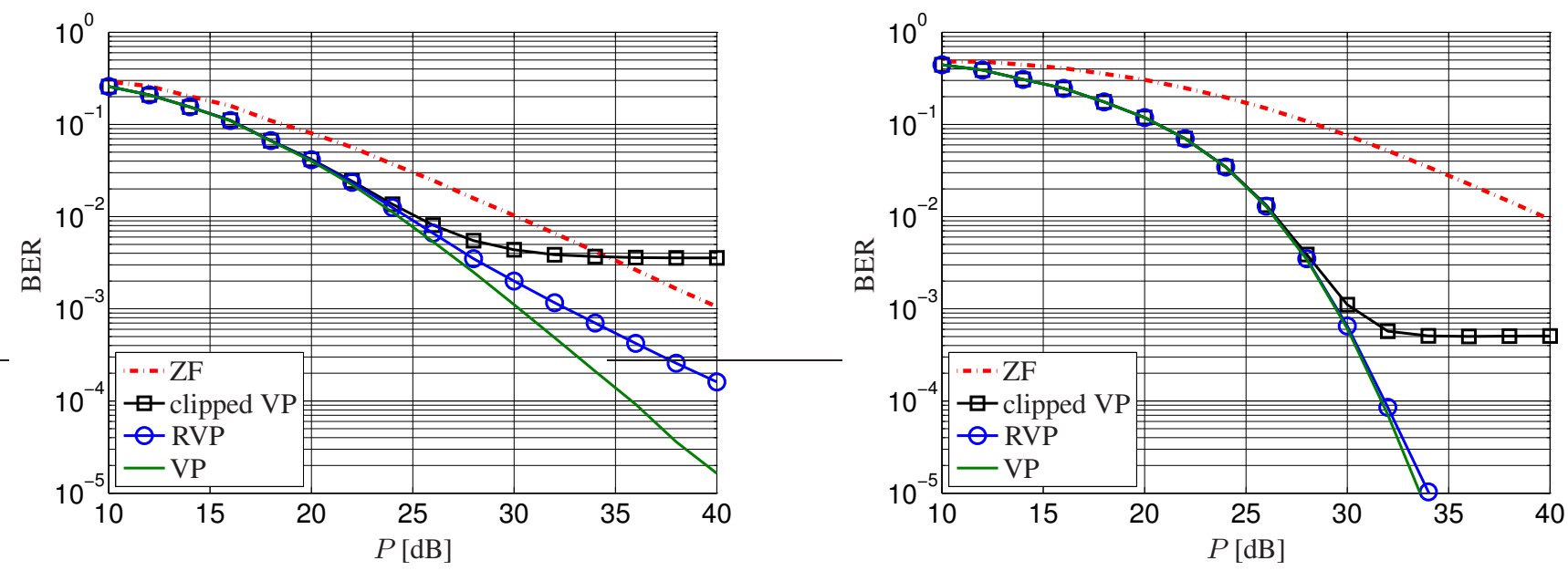

Fig. 1. BER versus SNR for conventional VP precoding for infinite dynamic range (labeled 'VP') and for finite dynamic range ('clipped VP'), ZF precoding (no perturbation), and RVP for $M=K=2$ (left-hand side) and $M=K=6$ (right-hand side).

$\mathcal{Z}^{K} \subset \mathbb{G}^{K}$ of perturbation vectors at the transmit side (cf. (7)):

$$
\tilde{\mathbf{z}}^{\star}=\arg \min _{\mathbf{z} \in \mathcal{Z}^{K}}\left\|\mathbf{H}^{\dagger}(\mathbf{s}+\tau \mathbf{z})\right\|^{2}
$$

We note that (11) can again be solved efficiently using the sphere encoder. Here, the finiteness of $\mathcal{Z}^{K}$ can potentially be exploited to reduce its complexity as compared to its application for solving the infinite lattice case associated with conventional VP (7). The set $\mathcal{Z}$ is chosen such that $\left|\operatorname{Re}\left\{z_{k}\right\}\right| \leq\left(\kappa-\left|\operatorname{Re}\left\{s_{k}\right\}\right|\right) / \tau$ and $\left|\operatorname{Im}\left\{z_{k}\right\}\right| \leq$ $\left(\kappa-\left|\operatorname{Im}\left\{s_{k}\right\}\right|\right) / \tau$ for all $z_{k} \in \mathcal{Z}$ and all $s_{k} \in \mathcal{A}$. This implies that no clipping occurs in the noise-free case and hence the probability for $r_{k}$ to be clipped vanishes asymptotically with increasing SNR. Consequently, RVP according to (11) avoids an error floor. However, the finite perturbation set may result in a somewhat larger power scaling factor, given by

$$
\tilde{\Gamma} \triangleq \min _{\mathbf{z} \in \mathcal{Z}^{K}}\left\|\mathbf{H}^{\dagger}(\mathbf{s}+\tau \mathbf{z})\right\|^{2} .
$$

Since the receiver noise variance equals $\tilde{\Gamma} / P$, the effective SNR of $\mathrm{RVP}$ is reduced. This effect will be the more pronounced the smaller the cardinality of $\mathcal{Z}$. We will next make these observations precise by studying the diversity order of RVP, defined as

$$
d \triangleq-\lim _{P \rightarrow \infty} \frac{\log \operatorname{Pr}\{\mathcal{E}\}}{\log P}
$$

Our main result is stated in the following theorem, whose proof is provided in the Appendix.

Theorem 1 For receivers with limited receiver dynamic range $\kappa$ (arbitrary but fixed), RVP precoding according to (3) and (11) under an instantaneous power constraint (2) achieves a diversity order of

$$
d_{\mathrm{RVP}}=M-K+1 .
$$

Discussion. First of all, the theorem shows that RVP does not suffer from an error floor. However, the diversity order achieved by RVP is the same as the one obtained with zero-forcing (ZF) precoding (i.e., channel inversion without perturbation). In fact, ZF precoding is a special case of RVP obtained with $\mathcal{Z}=\{0\}$. It is important to note, though, that in spite of identical diversity there may still be an SNR gap between RVP and ZF precoding. Indeed, our simulations indicate that RVP can perform close to optimum precoding (i.e., conventional VP without dynamic range limitation) over a wide range of SNRs before the small diversity order kicks in.
We are now also in the position to argue that (10) indeed holds true. According to [5], the optimum precoder achieves diversity order $M$. Since the theorem states that $d_{\mathrm{RVP}}<M$ for arbitrary $\kappa$ (remember that we assumed $K \geq 2$ ), it follows that optimum precoding requires an infinitely large perturbation set, or equivalently that the optimum precoder occasionally uses arbitrarily large perturbations, thereby verifying (10). Also, a large dynamic range is a consequence of attempting to approach optimal performance in terms of error probability.

\section{SIMULATION RESULTS}

We next present numerical results to assess the performance of conventional VP precoding with clipping and of RVP precoding. In all simulations, a 16-QAM symbol alphabet is used. Figure 1 shows bit error rate (BER) versus nominal SNR $P$ achieved by various precoders for two systems of dimension $M=K=2$ (on the left) and $M=K=6$ (on the right). In both cases, $d_{\mathrm{RVP}}=1$.

Conventional VP precoding with infinite dynamic range (labeled 'VP') is shown as the ultimate benchmark that cannot be achieved with limited dynamic range. ZF precoding (labeled 'ZF') is depicted as an extreme case of VP precoding with no perturbation $(\mathbf{z}=\mathbf{0})$. The other curves correspond to finite dynamic range and show conventional VP with clipping ('clipped VP') and RVP. The clipping level was chosen as $3 \tau / 2$, which allows RVP to use perturbations satisfying $\left|\operatorname{Re}\left\{z_{k}\right\}\right| \leq 1$ and $\left|\operatorname{Im}\left\{z_{k}\right\}\right| \leq 1$. This entails perturbation sets of size $81(K=2)$ and $531441(K=6)$.

The error floor of clipped VP is clearly seen for both systems. The much larger number of degrees of freedom in the system with $K=6$, however, entails a significantly lower error floor than with $K=2$. Furthermore, RVP outperforms conventional VP precoding with clipping. For $K=2$, the diversity $d_{\mathrm{RVP}}=1$ can be read in the plot, whereas for $K=6$ the high-SNR regime cannot be seen since it kicks in only for much larger (practically irrelevant) $P$. In the latter case, RVP performs within $0.5 \mathrm{~dB}$ of the VP precoding with infinite dynamic range over the whole BER range shown. This can be attributed to the fact that the perturbation set is large even though $\kappa$ is small. In contrast, the gap between RVP and VP precoding with infinite dynamic range is noticeable larger for $K=2$. This gap could be further reduced, however, by increasing the dynamic range and the size of the perturbation set. 


\section{CONCLUSION}

We showed that in case of receivers with limited dynamic range, conventional vector perturbation (VP) precoding suffers from an error floor. Hence, we proposed restricted VP (RVP) as an alternative that uses a finite set of perturbation vectors. RVP avoids the error floor but achieves a diversity order that is strictly less than that of the conventional VP with infinite dynamic range. However, our simulations indicated that the diversity loss is effective only at high SNRs, i.e., there is no significant performance gap for a wide range of SNRs, particularly for large system size.

While in this paper we restricted to perfect channel state information (CSI), our results can be generalized to the case of imperfect CSI (see [5]). Furthermore, our analysis also applies to the recently introduced transmit outage precoding scheme [8].

\section{APPENDIX: PROOF OF THEOREM 1}

By construction, detection errors in RVP occur only in the case of strong noise $v_{k}$. Since the variance of $v_{k}$ equals $\tilde{\Gamma} / P$, errors are likely to occur whenever $\tilde{\Gamma} \geq P$. If $\tilde{\Gamma} \leq P^{1-\epsilon}$ (for arbitrarily small $\epsilon>0$ ) the probability of error vanishes exponentially fast as $P$ tends to infinity. Thus, one may use the standard outage argument (cf. [9, Chapter 3] and [5]) to conclude that

$$
d=-\lim _{P \rightarrow \infty} \frac{\log \operatorname{Pr}\{\tilde{\Gamma} \geq P\}}{\log P} .
$$

It remains to show that the right-hand side of (13) equals $(M-K+1)$.

Let $\lambda$ and $\mathbf{u}$ denote the smallest eigenvalue and associated eigenvector, respectively, of $\mathbf{H} \mathbf{H}^{\mathrm{H}}$ and note that

$$
\lambda^{-1}\left|\mathbf{u}^{\mathrm{H}}(\mathbf{s}+\tau \mathbf{z})\right|^{2} \leq\left\|\mathbf{H}^{\dagger}(\mathbf{s}+\tau \mathbf{z})\right\|^{2} \leq \lambda^{-1}\|\mathbf{s}+\tau \mathbf{z}\|^{2} .
$$

As $\mathcal{Z}$ and $\mathcal{A}$ are finite, it follows that there exists a constant $c_{1}$, independent of $\mathbf{s}$ and $\mathbf{z}$, such that $\|\mathbf{s}+\tau \mathbf{z}\| \leq c_{1}$. The upper bound in (14) thus implies $\tilde{\Gamma} \leq \lambda^{-1} c_{1}$ and hence

$$
\operatorname{Pr}\{\tilde{\Gamma} \geq P\} \leq \operatorname{Pr}\left\{\lambda \leq c_{1} P^{-1}\right\} .
$$

As it holds that [10]

$$
-\lim _{P \rightarrow \infty} \frac{\log \operatorname{Pr}\left\{\lambda \leq c_{1} P^{-1}\right\}}{\log P}=M-K+1,
$$

we conclude

$$
-\lim _{P \rightarrow \infty} \frac{\log \operatorname{Pr}\{\tilde{\Gamma} \geq P\}}{\log P} \geq M-K+1 .
$$

The lower bound in (14) implies that $\tilde{\Gamma} \geq \lambda^{-1} f(\mathbf{u})$, where

$$
f(\mathbf{u}) \triangleq \min _{\mathbf{p} \in \mathcal{P}^{K}}\left|\mathbf{u}^{\mathrm{H}} \mathbf{p}\right|^{2}
$$

with $\mathbf{p} \triangleq \mathbf{s}+\tau \mathbf{z} \in \mathcal{P}^{K}$ and $\mathcal{P} \triangleq \mathcal{A}+\tau \mathcal{Z}$. As $\mathcal{P}$ is finite the minimum is guaranteed to exist. Hence, a sufficient condition for $\tilde{\Gamma} \geq P$ is given by

$$
\{f(\mathbf{u}) \geq \gamma\} \cap\left\{\lambda \leq \gamma P^{-1}\right\},
$$

for $\gamma>0$. Since $\mathbf{u}$ and $\lambda$ are independent [11], it follows that

$$
\operatorname{Pr}\{\tilde{\Gamma} \geq P\} \geq \operatorname{Pr}\{f(\mathbf{u}) \geq \gamma\} \operatorname{Pr}\left\{\lambda \leq \gamma P^{-1}\right\} .
$$

For this bound to be nontrivial, we need to find a $\gamma>0$ such that $\operatorname{Pr}\{f(\mathbf{u}) \geq \gamma\}>0$. To this end, note that $f(\mathbf{u})=0$ if and only if there is a $\mathbf{p} \in \mathcal{P}$ such that $\left|\mathbf{u}^{\mathrm{H}} \mathbf{p}\right|^{2}=0$. By the union bound it follows that

$$
\operatorname{Pr}\{f(\mathbf{u})=0\} \leq \sum_{\mathbf{p} \in \mathcal{P}} \operatorname{Pr}\left\{\left|\mathbf{u}^{\mathrm{H}} \mathbf{p}\right|^{2}=0\right\}=0 .
$$

Here, we used the fact that $\operatorname{Pr}\left\{\left|\mathbf{u}^{\mathrm{H}} \mathbf{p}\right|^{2}=0\right\}=0$ for any $\mathbf{p} \in \mathcal{P}$ since $\mathbf{u}$ is uniformly distributed over the unit sphere [11] (remember that $\mathcal{A}$ does not include 0 ).

Thus, there must be some $\overline{\mathbf{u}}$ for which $f(\overline{\mathbf{u}})>0$. Since $f(\mathbf{u})$ is continuous in $\mathbf{u}$, there exists $\gamma>0$ and a non-empty open neighborhood $\mathcal{U}$ of $\overline{\mathbf{u}}$ such that $f(\mathbf{u})>\gamma$ for all $\mathbf{u} \in \mathcal{U}$. Hence, $\operatorname{Pr}\{f(\mathbf{u}) \geq$ $\gamma\}=\operatorname{Pr}\{\mathbf{u} \in \mathcal{U}\}>0$. Using this $\gamma$ in (17) and applying (15) leads to

$$
-\lim _{P \rightarrow \infty} \frac{\log \operatorname{Pr}\{\tilde{\Gamma} \geq P\}}{\log P} \leq M-K+1 .
$$

Combining (13), (16), and (18) completes the proof.

\section{ACKNOWLEDGEMENT}

The authors thank Andreas Burg for drawing their attention to the dynamic range problem of vector perturbation precoding.

\section{REFERENCES}

[1] C. B. Peel, B. M. Hochwald, and A. L. Swindlehurst, "A vector-perturbation technique for near-capacity multiantenna multiuser communication - Part I: Channel inversion and regularization," IEEE Trans. Comm., vol. 53, no. 1, pp. 195-202, Jan. 2005.

[2] B. M. Hochwald, C. B. Peel, and A. L. Swindlehurst, "A vector-perturbation technique for near-capacity multiantenna multiuser communication - Part II: Perturbation," IEEE Trans. Comm., vol. 53, no. 3, pp. 537-544, March 2005.

[3] C. Windpassinger, R. F. H. Fischer, T. Vencel, and J. B. Huber, "Precoding in multiantenna and multiuser communications," IEEE Trans. Wireless Comm., vol. 3, no. 4, pp. 1305-1316, July 2004.

[4] R. F. H. Fischer, W.H. Gerstacker, and J.B. Huber, "Dynamics limited precoding, shaping, and blind equalization for fast digital transmission over twisted pair lines," IEEE Journal on Selected Areas in Communications, vol. 13, no. 9, pp. 16221633, Dec 1995.

[5] J. Jaldén, J. Maurer, and G. Matz, "On the diversity order of vector perturbation precoding with imperfect channel state information," in Proc. IEEE SPAWC'08, Recife, Brasil, July 2008, pp. 211-215.

[6] E. Agrell, T. Eriksson, A. Vardy, and K. Zeger, "Closest point search in lattices," IEEE Trans. Inf. Theory, vol. 48, no. 8, pp. 2201-2214, Aug. 2002.

[7] J. Jaldén and B. Ottersten, "On the complexity of sphere decoding in digital communications," IEEE Trans. Signal Processing, vol. 53, no. 4, pp. 1474-1484, Apr. 2005.

[8] J. Maurer, J. Jaldén, and G. Matz, "Transmit outage precoding with imperfect channel state information under an instantaneous power constraint," in Proc. IEEE SPAWC'08, Recife, Brasil, July 2008, pp. 66-70.

[9] D. Tse and P. Viswanath, Fundamentals of Wireless Communication, Cambridge University Press, 2005.

[10] L. Zheng and D. Tse, "Diversity and multiplexing: A fundamental tradeoff in multiple antenna channels," IEEE Trans. Inf. Theory, vol. 49, no. 5, pp. 1073-1096, May 2003.

[11] A. M. Tulino and S. Verdú, "Random matrices and wireless communications," Foundations and Trends in Communications and Information Theory, vol. 1, no. 1, June 2004. 